\title{
Ultrastructural Differentiation in the Distal Ends of Ameloblasts from the Presecretory Zone to the Early Secretory Zone
}

\author{
Toshihiko INAGE, Masaki FUJITA, Masatoshi KOBAYASHI, \\ Kouichi WAKAO, Masaharu KOBAYASHI, Noboru SAITO, \\ Hiroshi SHIBUYA, Masayuki SHIMADA and Kozo TAKAGI
}

(Received 2 June and accepted 6 June 1990)

Key words: ameloblasts, amelogenesis, dental enamel, dental enamel proteins, incisor, rats

\begin{abstract}
Morphological differentiation of the distal ends of ameloblasts (AMs) from the late presecretory to early secretory zones of the rat upper incisor was studied by electron microscopy.

Preameloblasts (PAs) showed a high columnar shape, with the nucleus located in the basal portion. The Golgi apparatus occupied the supranuclear region with type-1 vesicles, and microvilli were present at the distal cell membrane. Coarse-textured material was observed inside the type-1 vesicles and in the lateral intercellular spaces as well as along the distal cell membrane, whereas fine-textured material was found along the distal cell membrane. Near the region of initial enamel formation, large matrix islands were found in the lateral intercellular space. A thin electron-dense layer was observed on the dentin surface. This layer might have occurred as a result of diffusion after degradation of the coarse- or fine-textured material into a finer substance in the extracellular spaces. In the region of initial enamel formation, the distal cell membrane of AMs was flat, but shallow and narrow membrane invaginations were associated with the cell membrane close to the matrix islands. In the region of inner enamel formation, a cone-shaped Tomes' process was formed between large matrix islands which had developed in the intercellular spaces between the lateral portions of the distal ends of AMs. It was considered that the membrane invaginations which had existed at the distal end of PAs moved lower toward the distal terminal bar, thereafter becoming microvilli.
\end{abstract}

\section{Introduction}

AMs secrete enamel matrix during enamel formation and are also closely associated with its mineralization.

稲毛稔彦, 藤田正樹, 小林正俊, 若尾孝一, 小林政治, 斉藤登, 渋谷紘, 島田真幸, 高木幸三: Department of Anatomy, Nihon University School of Dentistry.

To whom all correspondence should be addressed: Dr. Toshihiko Inage, Department of Anatomy, Nihon University School of Dentistry, 1-8-13 Kanda-Surugadai, Chiyoda-ku, Tokyo 101, JAPAN. 
Since in order to perform this function AMs undergo a variety of morphological changes throughout their life cycle, ameloblastic differentiation from the presecretory stage to the secretory stage is of particular interest from a morphological viewpoint, and a number of electron microscopic studies have been reported $^{[1-11]}$. WARSHAWSKY et al. ${ }^{[12]}$ demonstrated that matrix islands appeared in the lateral intercellular spaces between the distal ends of secretory ameloblasts (SAs) and that their deposition caused cone-shaped Tomes' processes to form.

However, there has been a scarcity of studies on the morphological differentiation of the distal cytoplasm during the transition from the presecretory stage to the secretory stage, particularly with regard to the formation of microvilli under the distal terminal bars of SAs and the secretory sites of the enamel matrix ${ }^{[4]}$.

In the present study, therefore, serial observations of the distal end of AMs in the rat incisor from the late presecretory stage to the early secretory stage were conducted by electron microscopy.

\section{Materials and Methods}

Twelve male Wistar rats each weighing about $40 \mathrm{~g}$ were used. Under Nembutal anesthesia, each animal was perfused from the left ventricle with $2.5 \%$ glutaraldehyde solution including $2 \%$ paraformaldehyde diluted with $0.08 \mathrm{M}$ cacodylate buffer ( $\mathrm{pH}$ 7.4). After decalcification in 0.1 M EDTA ( $\mathrm{pH} 7.4$ ) for three weeks, the upper incisors were cut transversely into five blocks and each of the divided blocks was further cut mid-sagittally with a razor blade. They were then post-fixed in $1 \% \mathrm{OsO}_{4}$. After dehydration in ethanol, the specimens were embedded in Araldite. The tissues were cut into sections about $1 \mu \mathrm{m}$ thick, then stained with toluidine blue. After staining, the transition from the presecretory zone to the early secretory zone was identified by light microscopy and the same zone was cut into ultrathin sections. The ultrathin sections were double-stained with uranyl acetate and lead citrate, and then observed with a Hitachi H-800 electron microscope.

\section{Results}

1. Late presecretory zone

PAs showed a high columnar shape, a nucleus located in the basal portion, and mitochondria accumulated at the basal end. The Golgi apparatus occupied the supranuclear region. A terminal bar was evident at the distal end, and microvilli were found at the distal cell membrane. No rough endoplasmic reticulum was found in the cytoplasm under the distal terminal bar, but a number of vesicles and coated vesicles with various diameters were present. The type-1 vesicles ${ }^{[4]}$ were located near the Golgi apparatus and in the distal cytoplasm (Fig. 1).

Coarse-textured material ${ }^{[4]}$ was observed inside the type-1 vesicles, in the lateral intercellular spaces between the PAs and along the distal cell membrane. A material corresponding to fine-textured material ${ }^{[4]}$ was observed in the extracellular spaces under the distal cell membrane. In the invaginated area of the microvilli, collagen fibrils were observed (Fig. 2). Narrow membrane invaginations were apparent at the distal cell membrane, and collagen fibrils were recognized inside them (Fig. 3). 
In the area closer to the region of initial enamel, the type-1 vesicles became larger in both size and number, whereas near the Golgi apparatus an increase in the number of spherical bodies ${ }^{[13]}$ was observed (Fig. 4). Small matrix islands were present in the lateral intercellular spaces between the PAs. The microvilli at the distal end gradually disappeared, and the distal cell membrane became flat (Figs. 4-6). An electron-dense layer was evident on the surface of the dentin (Figs. 4-6).

Furthermore, the width of the electron-dense layer became progressively thicker toward the region near the zone of initial enamel formation, and its border facing the dentin invaded into the dentin along the collagen fibrils. Material considered to be enamel precursor was observed immediately under the cell membrane of PAs (Fig. 6).

2. Initial enamel secretory zone

A thin layer of initial enamel began to appear immediately under the distal ends of SAs. Although the number of type-1 vesicles decreased, there were many granules within the cells and the rough endoplasmic reticulum was developed conspicuously (Fig. 7). A large number of secretory granules were observed in the distal cytoplasm (Fig. 8), and coated pits and shallow and narrow membrane invaginations were observed at the distal cell membrane (Figs. 8, 9).

The amount of cytoplasm under the distal terminal bar was increased where initial enamel about $1 \mu \mathrm{m}$ thick had formed (Fig. 10). A large amount of enamel matrix was observed in the lateral intercellular spaces between the distal ends of SAs, particularly near the membrane invaginations (Fig. 11). The thickness of the initial enamel was not even, with a discontinuous portion due to the cytoplasm of SAs (Figs. 11, 12). In the cells which formed cone-shaped Tomes' processes at the distal end of SAs, microvillus-like membrane invaginations were observed in the lateral membrane of the Tomes' processes (Figs. 11, 12, arrows). In the region near the site of inner enamel formation, a large number of secretory granules was present in the cytoplasm at the distal end (Fig. 13).

3. Inner enamel formation zone

The cytoplasm at the distal end was fully developed and formed Tomes' processes. Microvilli were observed in the lower part of the terminal bar, and the cell membrane was invaginated from the apex of the Tomes' processes (Fig. 14).

\section{Discussion}

In the early stage of tooth formation, PAs and the dentin are divided by a continuous basal lamina, which eventually disappears when enamel formation has started, as clarified by many electron microscopic studies ${ }^{[1-11]}$. $\mathrm{K}_{\mathrm{ALLENBACH}}{ }^{[4]}$,

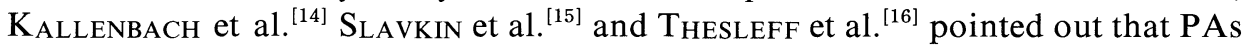
undergo morphological differentiation to SAs upon transmission of a stimulus from the mesenchyme to the epithelium via ectomesenchymal contacts. On the basis of an electron microscopic study of rat molars, REITH ${ }^{[1]}$ suggested that after the basal lamina had been resorbed by PAs, stippled material destined to become enamel was secreted. $\mathrm{K}_{\mathrm{ALLENBACH}}{ }^{[4]}$ and $\mathrm{K}_{\mathrm{ASAMO}}{ }^{[11]}$, studying rat incisors, reported that before removal of the basal lamina, coarse-textured material (corresponding to the stippled material) was observed. Then, after the disappearance of the 
basal lamina, the coarse-textured material was replaced by fine-textured material. In the present study, both coarse- and fine-textured materials were observed in the lateral intercellular spaces between the PAs at the distal end in the region where the basal lamina had disappeared, and a zone with an electron-dense layer was present on the dentin surface. $\mathrm{K}_{\text {ALLENBACH }}{ }^{[4]}$ and $\mathrm{K}_{\text {ASAMO }}{ }^{[11]}$ also described that both the coarse- and fine-textured materials present in the dentin always showed a cluster-like form. The above-mentioned electron-dense layer might occur by diffusion of the coarse-textured material following degradation into smaller-sized material extracellularly after secretion from the PAs.

The synthetic pathway of the textured material is not yet known in detail. The coarse-textured material was found in type- 1 vesicles, which were present near the Golgi apparatus and in the distal cytoplasm when the coarse-textured material appeared in the intercellular spaces. This suggests that type-1 vesicles are related to the synthesis and secretion of the coarse-textured material.

WARSHAWSKY et al. ${ }^{[12]}$ observed that small globules appeared in the lateral intercellular spaces between the distal portions of PAs after disappearance of the basal lamina. These then grew into large matrix islands, finally becoming the initial enamel. The same result was obtained in the present study; that is, with the appearance of the matrix islands, enamel was formed under the PAs. It is therefore possible to consider that the matrix islands are precursors of the enamel which might be secreted by SAs. The matrix islands were always observed near the membrane invaginations of the PAs. This result suggests that the membrane invaginations might be the site of secretion of the enamel matrix in PAs.

As for the ultrastructural changes in the distal cell membrane from PAs to SAs, matrix islands appeared in the lateral intercellular spaces between the PAs in the distal end, near the region of initial enamel formation. The membrane invaginations were evident only in this location. As enamel formation progressed further, the matrix islands gradually increased in size, they pressed the distal ends from a lateral direction, finally forming cone-shaped Tomes' processes at the distal end of the SAs. Since at this time the distal apex cytoplasm is still growing toward the dentino-enamel junction, the apex of the Tomes' processes extends distally. The membrane invaginations which were previously located at the distal end of the PAs then become located in the lower part of the distal terminal bar. Consequently, the membrane invaginations previously present at the distal end are observed as microvilli by electron microscopy.

\section{References}

[1] Rerth, E.J.: The early stage of amelogenesis as observed in molar teeth of young rats, $J$. Ultrastruct. Res., 17, 503-526, 1967

[2] Elwood, W.K. and Bernstein, M.H.: The ultrastructure of the enamel organ related to enamel formation, Am. J. Anat., 122, 73-94, 1968

[3] MoE, H.: Morphological changes in the infranuclear portion of the enamel-producing cells during their life cycle, J. Anat., 108, 43-62, 1971

[4] Kallenbach, E.: Electron microscopy of the differentiating rat incisor ameloblast, $J$. Ultrastruct. Res., 35, 508-531, 1971

[5] Silva, D.G. and KaILIs, D.G.: Ultrastructural studies on the cervical loop and the develop- 
ment of the amelo-dentinal junction in the cat, Archs. Oral Biol., 17, 279-289, 1972

[6] Katchburian, E. and Holt, S.J.: Studies on the development of ameloblasts. I. Fine structure, J. Cell Biol., 2, 415-447, 1972

[7] Kallenbach, E.: Fine structure of differentiating ameloblasts in the kitten, Am. J. Anat., 145, 283-318, 1976

[8] Matthiessen, M.E. and Rømert, P.: Ultrastructure of the human enamel organ. I. External enamel epithelium, stellate reticulum, and stratum intermedium, Cell Tissue Res., 205, 361-370, 1980

[9] Matthiessen, M.E. and RøMert, P.: Ultrastructure of the human enamel organ. II. Internal enamel epithelium, preameloblasts, and secretory ameloblasts, Cell Tissue Res., 205, 371-382, 1980

[10] Skobe, Z., Stern, D. and Prostak, K: Ultrastructure of differentiating preameloblasts from tooth germs of the permanent dentition of Macaca mulatta and Macaca arctoides, Calcif. Tissue. Int., 33, 603-618, 1981

[11] Kasamo, T.: An electron microscopic mapping from cervical loop to secretory zone of rat ameloblasts, Nihon Univ. Dent. J., 59, 543-569, 1985 (in Japanese)

[12] Warshawsky, H. and Vugman, I.: A comparison of the protein synthetic activity of presecretory and secretory ameloblasts in rat incisors, Anat. Rec., 188, 143-172, 1977

[13] Walton, R.E and Eisenmann, D.R.: Ultrastructural examination of various stages of amelogenesis in the rat following parenteral fluoride administration, Arch. Oral Biol., 19, 171-182, 1974

[14] Kallenbach, E. and Piesco, N.P.: The changing morphology of the epithelium-mesenchyme interface in the differentiation zone of growing teeth of selected vertebrates and its relationship to possible mechanisms of differentiation, J. Biol. Buccale, 6, 229-240, 1978

[15] Slavkin, H.C., Trump, G.N., Brownell, A. and Sorgente, N.: Epithelial-mesenchymal interactions, mesencyhmal specificity. In Cell and Tissue Interactions, Lash, J.W. and Burger, M. M., Eds., Society of General Physiologists Series, 32, Raven Press, New York, 29-46, 1977

[16] Thesleff, I., Lehtonen, E., Wartiovaara, J. and Saxen, L.: Interference of tooth differentiation with interposed filters, Dev. Biol., 58, 197-203, 1977 
Fig. 1 PA. Nucleus (N) is located in the basal portion and mitochondria (M) are accumulated in the basal portion. Microvilli (arrows) have developed at the distal end. The Golgi apparatus (G) occupies the supranuclear region, along with type-1 vesicles (T1). D: dentin $x 2,700$

Fig. 2 Higher-power magnification of the distal ends of the PA in the same region as in Fig. 1. The distal cytoplasm includes a tubular structure (arrow) and a type-1 vesicle (T1). Coarse-textured material (CTM) is observed in the lateral intercellular space and along the distal cell membrane. FTM: fine-textured material. $\quad \times 12,000$

Fig. 3 High-power magnification of the distal ends of PAs located near the initial enamel formation zone. Microvilli at the distal end gradually disappear but shallow and narrow membrane invaginations (arrows) start to appear. $\quad$ x 12,000

Fig. 4 PAs in the region closest to the initial enamel formation zone. The number and size of type-1 vesicles (T1) are increased. Electron-dense layer is evident on the surface of the dentin (arrows). D: dentin; G: Golgi apparatus; MI: matrix island; N: nucleus; SP: spherical body. $\quad$ x3,600

Figs. 5, 6 Distal ends of PAs in the same region. Arrows: electron-dense layer of the dentin. MI: matrix islands. $\quad \mathrm{x} 12,000$

Fig. 7 The SAs involved in initial enamel formation. Initial enamel (IE) is evident on the dentin surface. N: nucleus; G: Golgi apparatus; MI: matrix island; SP: spherical body. x5,000

Figs. 8,9 Higher-power magnification of the distal ends of SAs at the same region as in Fig. 7. A large number of secretory granules (arrows) are present in the distal end of the cytoplasm but type-1 vesicles are not found. Arrow heads: membrane invagination; IE: initial enamel; MI: matrix islands. $\mathrm{x} 12,000$

Fig. 10 SAs in the region of formation of initial enamel (IE) approximately $1 \mu \mathrm{m}$ thick. D: dentin; DTB: distal terminal bar; G: Golgi apparatus; N: nucleus. $\quad$ x5,000

Figs. 11, 12: The distal ends of SAs at the same region as in Fig. 10. Membrane invaginations (arrows) are present at the cell membrane near the matrix islands (MI). In Fig. 12, the distal cell membrane of SAs connects directly with the dentino-enamel junction. D: dentin; IE: initial enamel. $\times 5,000$

Fig. 13 The region adjacent to that of inner enamel formation. A cone-shaped process is formed at the distal end of a SA. D: dentin; E: enamel; Arrows: secretory granule x9,000

Fig. 14 Inner enamel formation zone. A Tomes' process is formed at the distal end. Arrows: secretory granule; E: enamel; Mm: membrane invagination; MV: microvilli.

$\mathrm{x} 9,000$ 


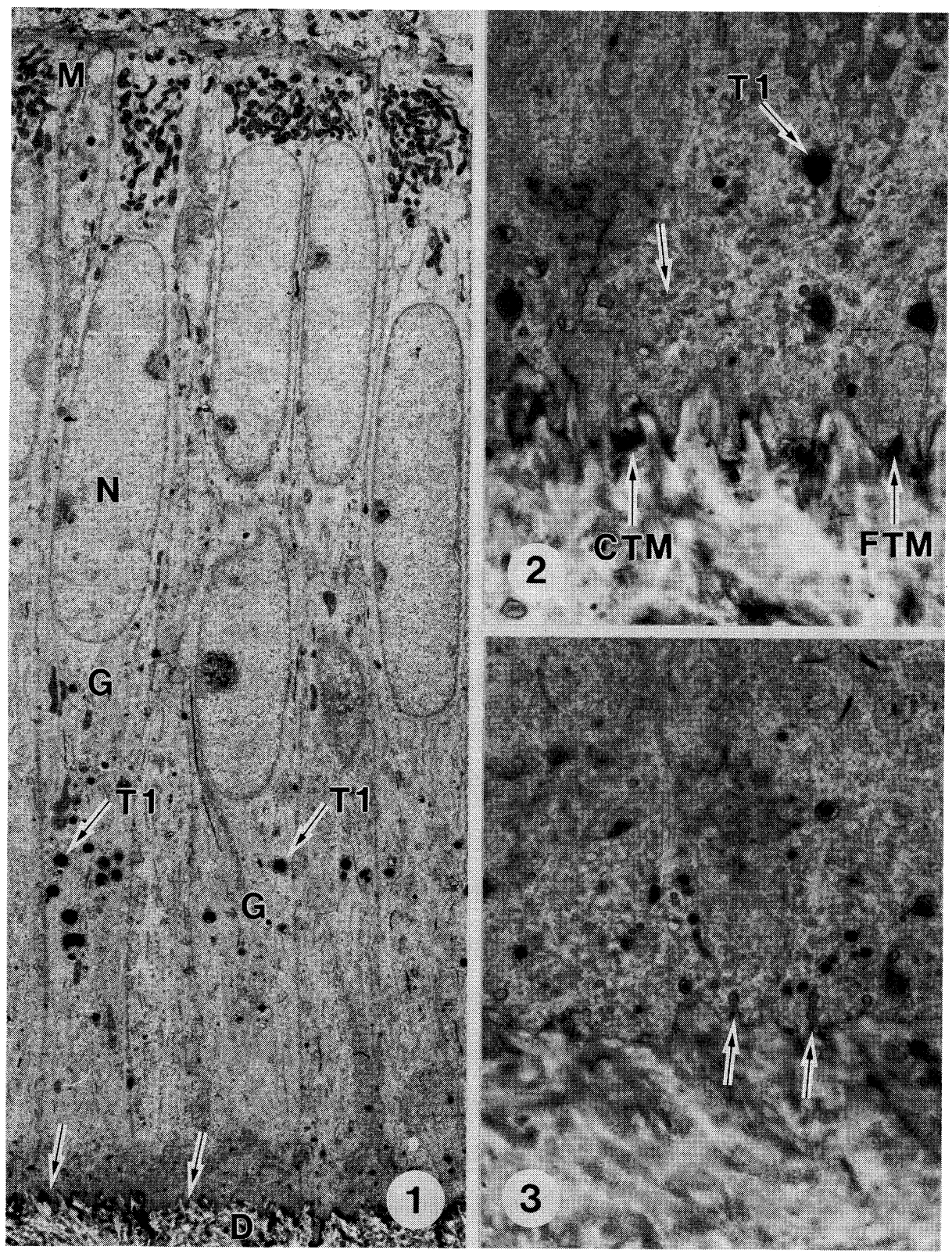




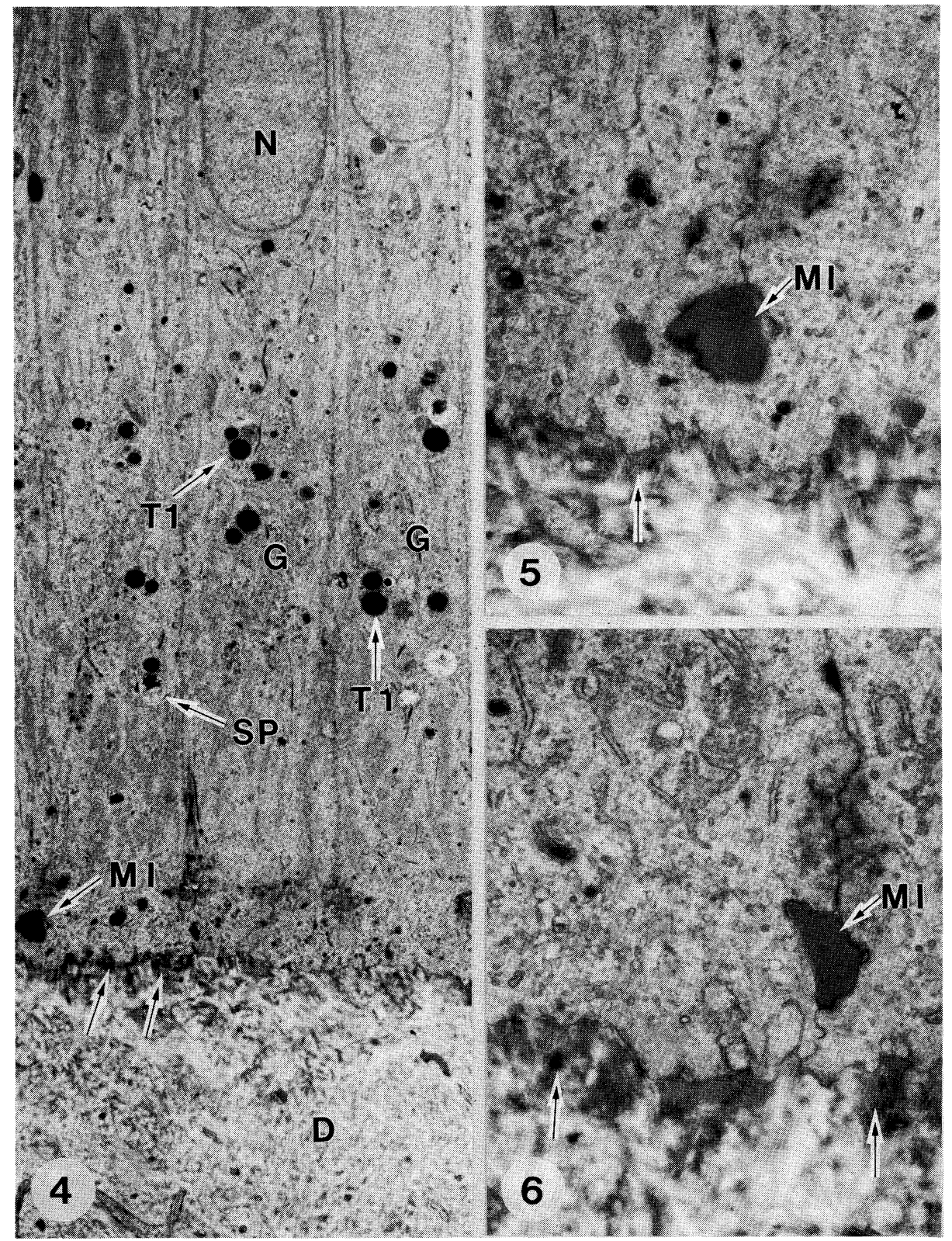



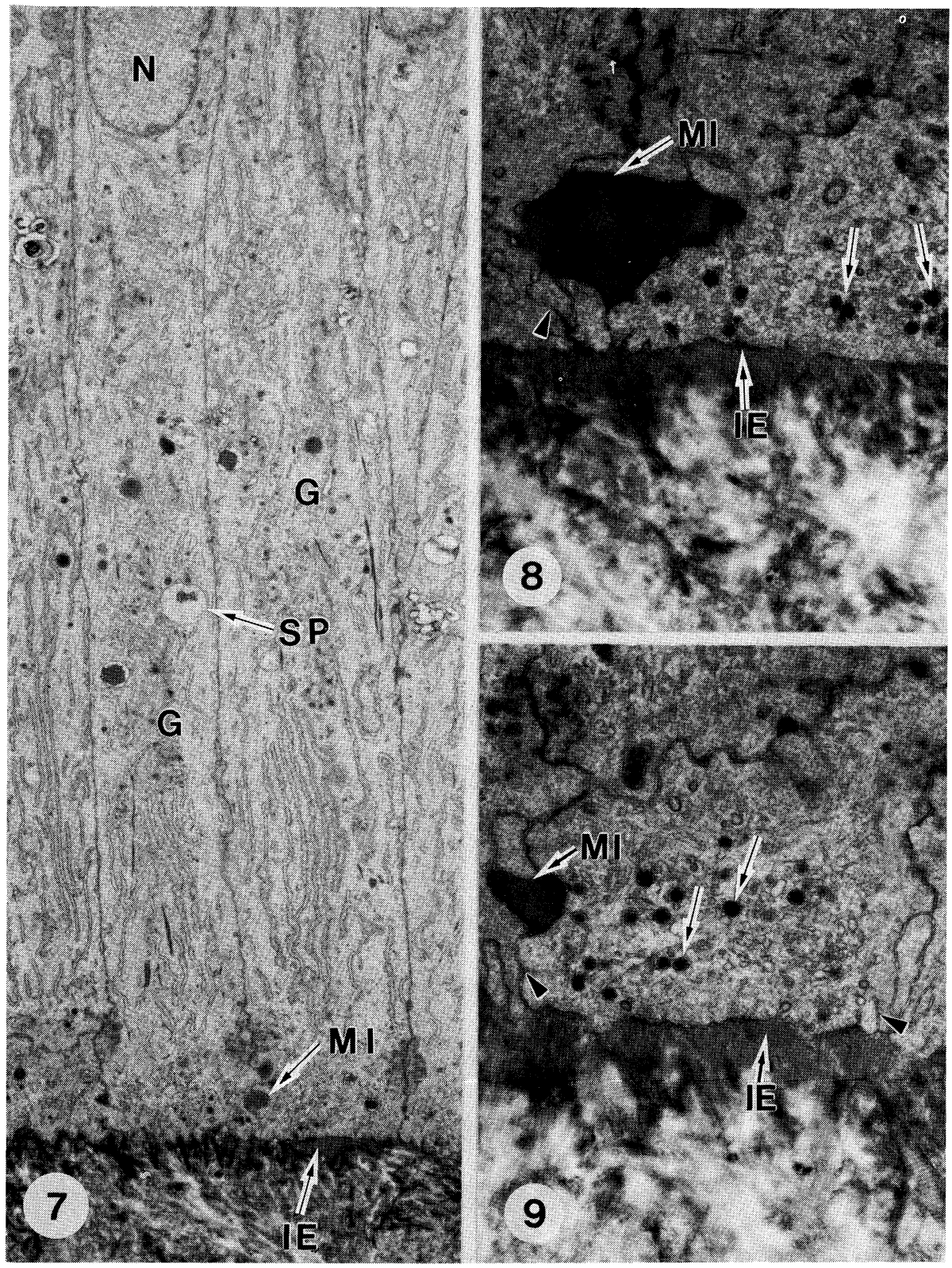


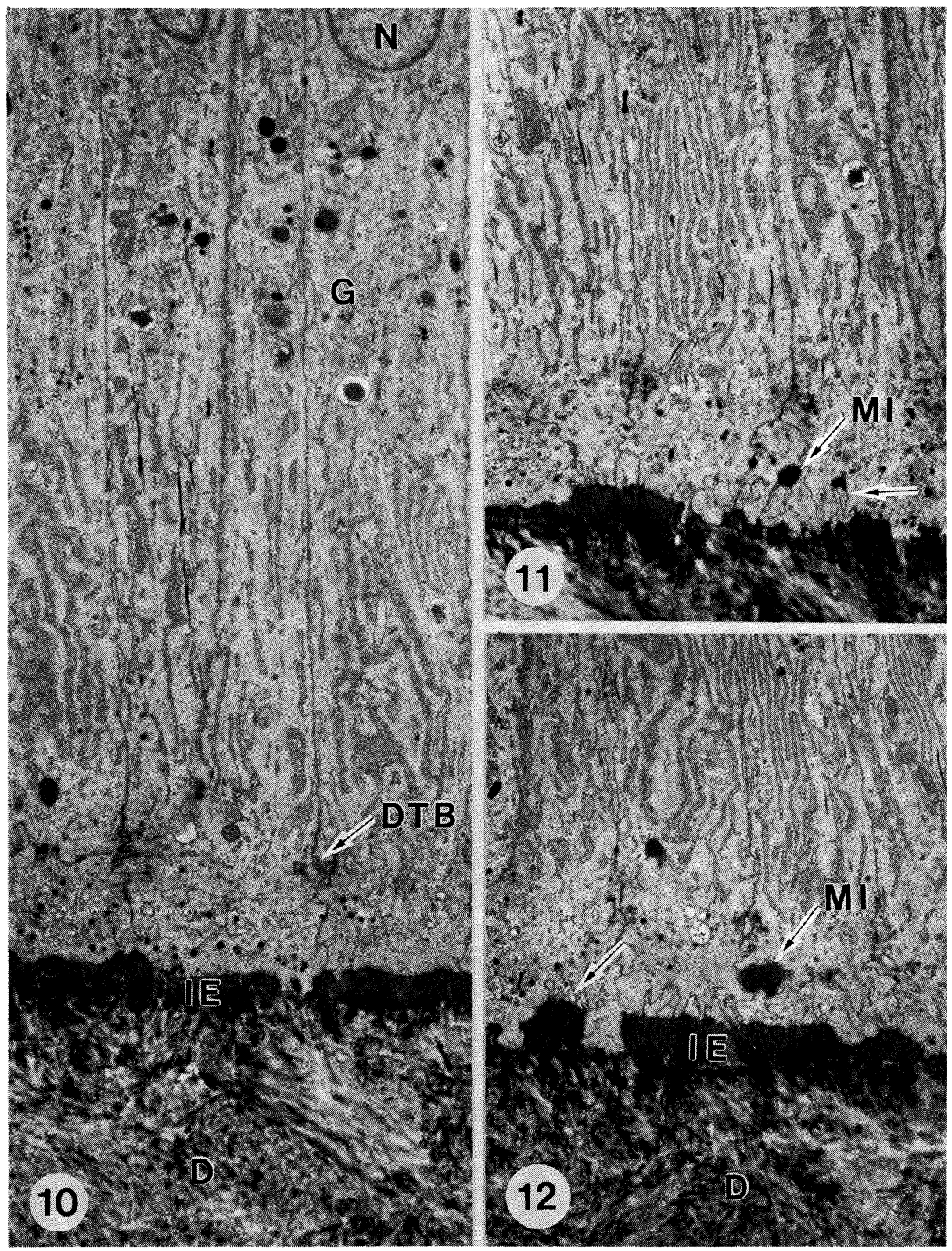




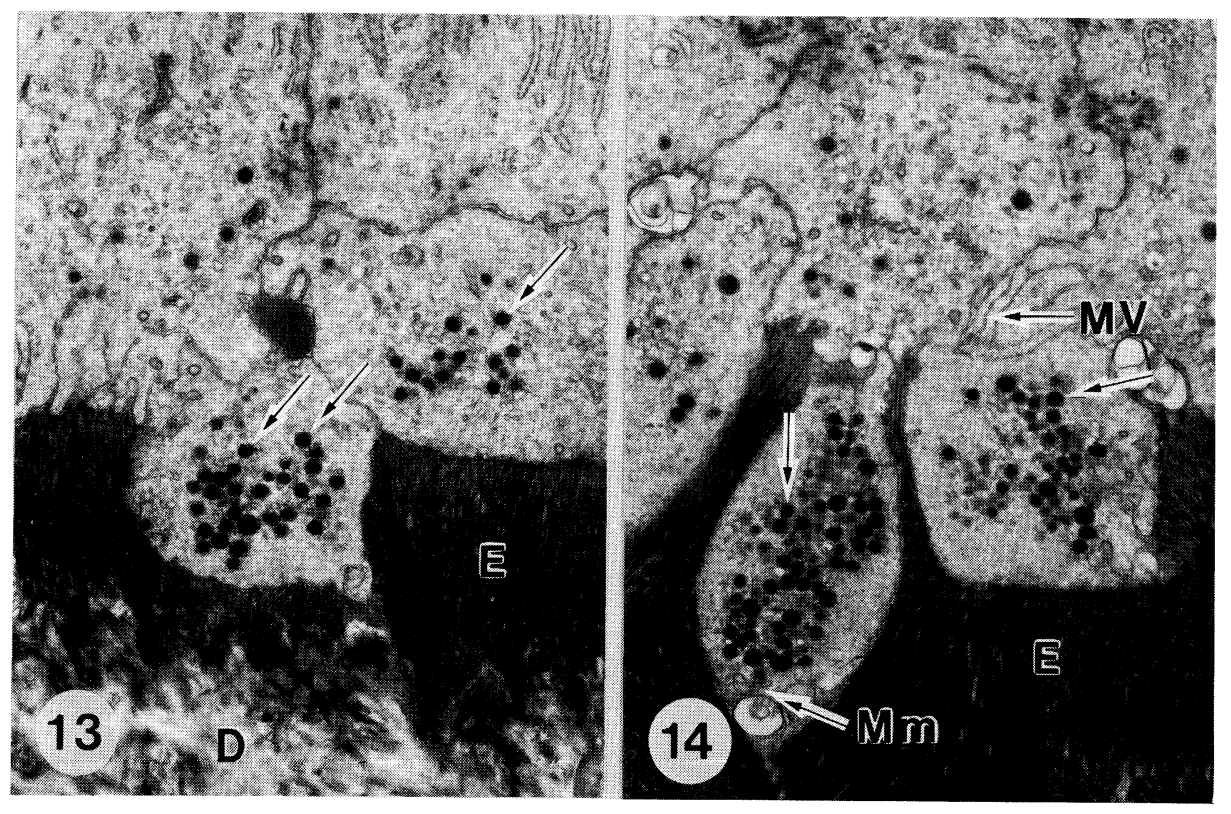

